\title{
Control system of automatic garage using programmable logic controller
}

\author{
K. Selvakumar, R. Palanisamy, Patti Nikhil, D. Karthikeyan, D. Selvabharathi \\ SRM Institute of Science and Technology, India
}

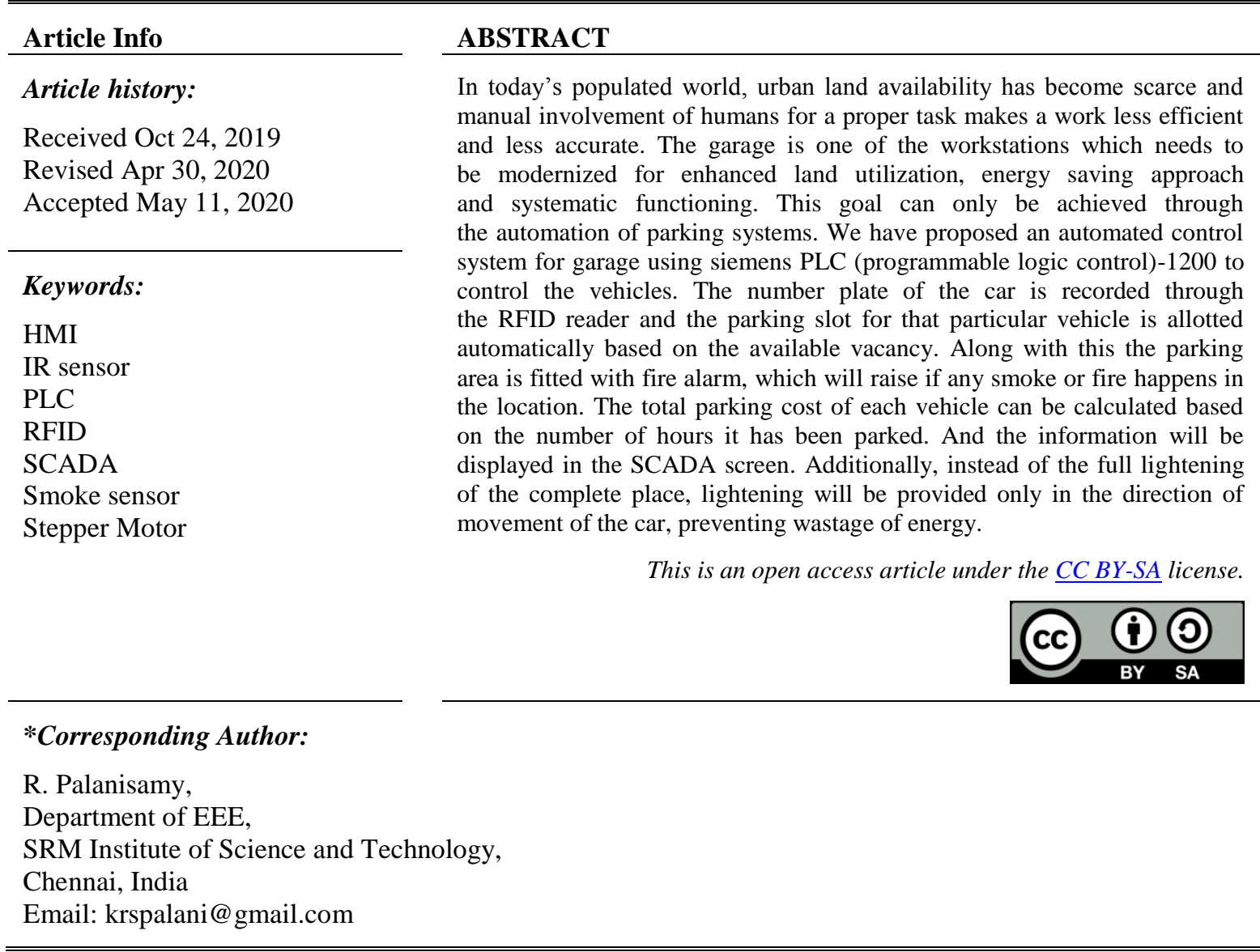

\section{INTRODUCTION}

PLC (programmable logic controller): PLC is an industrial digital computer which has been designed for the control of automated devices such as industrial process, manufacturing processes, robotics, assembly line and control applications. PLC's were designed to replace hard wired relays as they are easy to program and has high reliability [1-5]. It is a real time device which is designed to produce responses to input data within a limited time. The PLC kit consists of input modules, output modules, CPU (central processing unit), rack assembly, timer, counter, memory and programming unit. It has 14 digital input and 10 digital output and 2 analog input and 1 analog output [6]. PLC uses five types of programming languages which are: ladder logic, sequential function charts, functional block diagram, structured list, and instruction list. The ladder logic is the most commonly used PLC programming language. The power supply given to the PLC is $24 \mathrm{~V}$ [7-10].

SCADA is a system software for a process control. SCADA is a central control system which consists of controller network interfaces, input-output communication and software [11-16]. SCADA is used to control and monitor all industrial, manufacturing, infrastructural, production and development processes. It's used in for automation so minimal labour work is required upon its application. It is a centralized system 
used to wire and wireless technologies [17]. The components of SCADA are sensors, RTU (remote terminal units). The functions of SCADA system are data acquisition, data communication, data presentation, monitoring and control $[18,19]$.

RFID uses electromagnetic fields to automatically identify and track tags attached to objects. The RFID consists of a transmitter and a receiver. The receiver sends electromagnetic signals to the card and sends it to the coil. The coil generates signals to EPROM [20]. The EPROM again sends back the signals to the receiver. This process of sending it back is called Demodulation. This is then send it back to the required device via serial port. There are 2 types of RFID. They are passive and active RFID. The Stepper Motor is a brushless DC motor that divides a full rotation into an equal number of steps. Its rotor rotates through a fixed angular step in response to each input current pulses receive by its controller [21].

Stepper motor driver is a circuit or device that provides the necessary current and voltage to a stepper motor so that it has a smooth operation. It is a circuit that applies a voltage to any of the four stator coils of the motor. Driver can be built with IC such as ULN2003, four Darlington transistors or four power transistors [6]. The LPC2148 microcontroller is a 16-bit or 32-bit microcontroller based on ARM7 family. It is designed with several in-built features \& peripherals. The on-chip static RAM is $8 \mathrm{kB}-40 \mathrm{kB}$, and the on-chip flash memory is $32 \mathrm{kB}-512 \mathrm{kB}$. It has one or two 10-bit ADCs [22]. The working voltage is 5 volts. The LED is a light source which uses semiconductors and electroluminescence to create light. In other words, light-emitting diode (LED) is a semiconductor device that emits light when an electric current is passed through it. Light is produced when the particles that carry the current (known as electrons and holes) combine together within the semiconductor material [23].

An infrared sensor is an electronic device that emits in order to sense some aspects of the surroundings. An IR sensor can measure the heat of an object as well as detects the motion. These types of sensors measures only infrared radiation, rather than emitting it that is called as a passive IR sensor. Usually in the infrared spectrum, all the objects radiate some form of thermal radiations [24]. The emitter is simply an IR LED (light emitting diode) and the detector is simply an IR photodiode which is sensitive to IR light of the same wavelength as that emitted by the IR LED. When IR light falls on the photodiode, the resistances and these output voltages, change in proportion to the magnitude of the IR light received [25].

A relay is an electromagnetic switch operated by a relatively small electric current that can turn on or off a much larger electric current. Relays can work either as switches (turning things on and off) or as amplifiers (converting small currents into larger ones). To work as an amplifier, we give the operating voltage at once end of the relay coil. A transistor is connected to the other end of the relay coil. The voltage that has to be amplified is given to the base of a transistor due to which the circuit gets completed from collector to emitter. And hence the voltage we give at the com point of relay (i.e. the amplified voltage we want), is available at the NC point of the relay.

\section{PROPOSED AUTOMATIC GARAGE CONTROL SYSTEM}

The hardware comprises PLC (programmable logic control) Siemens S7-1200 kit, LPC2148 microcontroller, infrared sensor, smoke sensor, RFID [radio frequency identification], LEDs and stepper motor. Additional hardware components used are transformer and rectifier. The software part includes PLC for building ladder logics and SCADA screen is used for displaying. IR sensors are used to detect the presence of a vehicle in various slots within the infrared range, which will be further sent to the relays. each slot is equipped with ir sensor for the detection of vehicles. Smoke sensors senses smoke, typically as an indicator of fire. A single smoke sensor is used for a range of four slots for the detection of smoke and thus fire, which is shown in Figure 1.

The signal is not sent directly to the PLC as PLC can detect input voltages in the range of $24 \mathrm{~V}$ only. Hence from the relay signal is given to the PLC. Here, the relay circuit boosts the voltage for the preferred use. There are 2 RFID's in the project. One at the entrance and the other at the exit side. The data of RFID is analog data. Hence, the data from the RFID is first fed to the microcontroller (LPC2148) which analyses it and then gives signal to the PLC. For further processes. The PLC kit is programmed through the ladder logic for specific operations. The ladder logic designing consists of three parts:

- Main logic: connecting the LED's, RFID's, Motor's and the sensors to work with respect to each other.

- RFID logic: both the RFID's are connected and designed for comparison of values and flowingly they give the output.

- Motor logic: both the motors are designed in a way that the gates open when there is an absence of slot in the parking area.

- Car parking: if there is space then only allotment of slots takes place otherwise gate doesn't open and the LED's glow in the direction of movement of the car. 


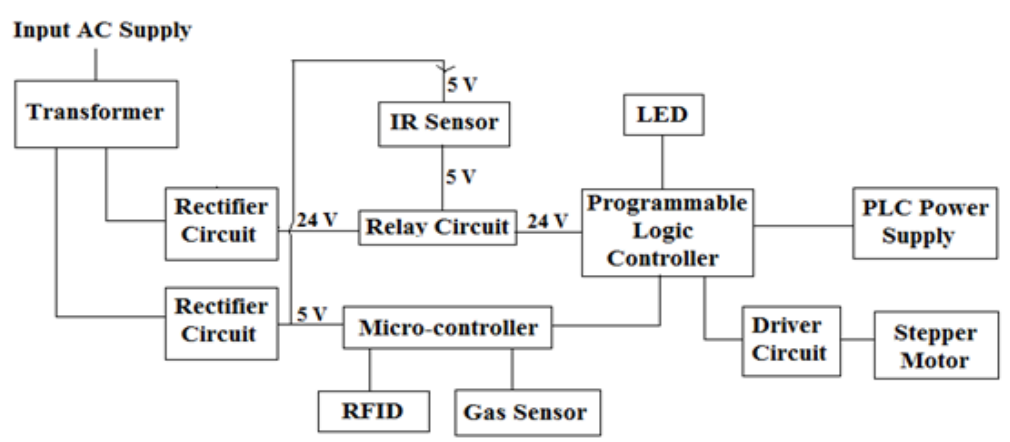

Figure 1. Block diagram of proposed system

\section{PROGRAMMABLE LOGIC CONTROLLER [PLC]}

A PLC is a small digital computer which is used to control the manufacturing processes with high reliability, which is shown in Figure 2. A PLC is useful because of it is ease of programming and ease of fault diagnosis. It is used as automation controllers in harsh environments. It is a hard-real time device which produces output signals with respect to input signals within limited time. The basic PLC hardware architecture consists of power supply, input module, CPU programming, output module, field inputs/control elements process machines. A power supply of $24 \mathrm{~V}$ is required for the application of PLC kit. The PLC can run with SMPS or without SMPS. If it is with SMPS then the voltage is $230 \mathrm{~V}$ AC and varied output DC can be generated. If it is without SMPS then direct $24 \mathrm{~V}$ from direct DC supply is supplied. The input output modules can be subdivided into compact type or standard type. In compact type extra modules can not be added whereas in standard type more number of modules can be added. Based on Input and Output expansions it can further be divided into Mini OR Micro, Medium and Rack Type. In the mini type only 3-4 modules can be added. In the medium type 8 modules can be added and in the rack type 32 modules can be added. It has 14 input-digital pins and 10 input-digital pins. It also has 2 analog-input pins and 1 analog-output pin.

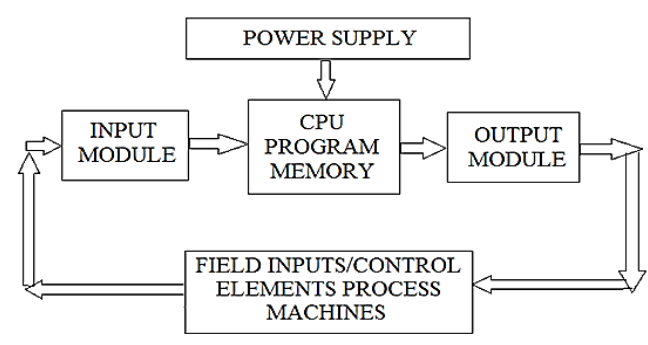

Figure 2. Basic PLC architecture

In Figure 3 shows P1I stands for process image input and PIQ stands for process image output. The function of a timer is that it keeps the output on for a certain length of time. The timers used in PLC are T-ON, T-OFF, TP. The T-ON timer switches on after a certain time specified. The T-OFF timer switches off after certain time and the TP remains on for a certain period. Counters are used for counting items. The evalution of PLC is shown in Table 1. The common types of counters are up counters, down counters and up/down counters, which is shown in Figure 4.

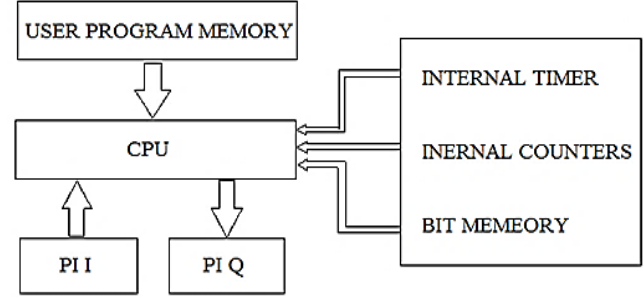

Figure 3. Basic block diagram

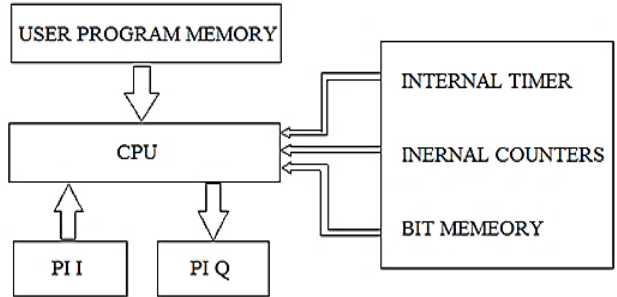

Figure 4. Memory unit 


\begin{tabular}{|c|c|c|}
\hline PLC VERSIONS & TYPE & \\
\hline LOGO name Changed to SMART LOGO & MINI & Nano-Type PLC \\
\hline S7-200 & MINI & Nano-Type PLC \\
\hline S7-1200 & MICRO & Voltage Level/Application Oriented \\
\hline S7-300 & INDUSTRIAL & Application Rack Type PLC \\
\hline S7-1500 & INDUSTRIAL & For all applications \\
\hline S7-400 & INDUSTRIAL & Same as S7-300, High Memory \\
\hline
\end{tabular}

\section{RFID}

RFID is a device which captures the digital data in RFID tags via radio frequency of radio waves. It is similar to barcode reader. However, RFID stands out from Barcode Reader in the fact that RFID reads data outside the line of sight whereas Barcode Reader only reads in the aligned preference with respect to the object through a scanner. So, RFID identify objects, collects data and helps and feeds those data to the computer systems without physical contact for their respective work. When an RFID tag passes through the RFID reader, it detects the activation signal from the antenna, which is shown in Figure 5. An RFID comprises of RFID tag, RFID reader and a processor/controller. An RFID tag consists of integrated circuit and an antenna. The tag is shielded with protective materials (such as plastics) to hold it together and prevent damages in environmental conditions. There are two types of RFID systems. They are:

- Active RFID system: in this system, the tags have their own power source i.e., external source. This can be used for high range applications.

- Passive RFID systems: in this system, the tag is powered via the reader antenna to the tag antenna. This is used for short range transmission.

In this project we are using two RFID's i.e., one for entry level for detection of the vehicle RFID number and the other for the exit level for noting down the outgoing vehicle. Through this process, the parking time could be calculated and thus the parking fee could also be calculated. Two analog pins of the PLC kit is used for the process. RFID_1 uses IW64 and RFID_2 uses IW66 as the analog inputs. The analog values will have to be converted into integer. As the rated range for PLC is $10 \mathrm{~V}$ which is similar to integer value of 27648 . So, 27648 is the analog rated maximum value. RFID calculation:

$$
10 \mathrm{~V}=27648 \text { (Constant) }
$$

As the output voltage received from the RFID is $3 \mathrm{~V}$. So, the $3 \mathrm{~V}$ is divided among 8 output on basis of its phase rotation of motor.

$$
\begin{aligned}
& 3 \mathrm{~V} / 8=0.375 \mathrm{~V} \\
& 0.375 \mathrm{~V}=(0.375 * 27648) / 10=10368 \text { (constant value) }
\end{aligned}
$$

These constant values are used during the initialization of RFID's by assigning it to the comparators to compare. For example, the constant value of Car_1 is 1023 so, it lies within a range of 950 and 1070. Similarly, for all the Car's the values should be assigned so that that the comparison could take place, which is shown in Table 2.

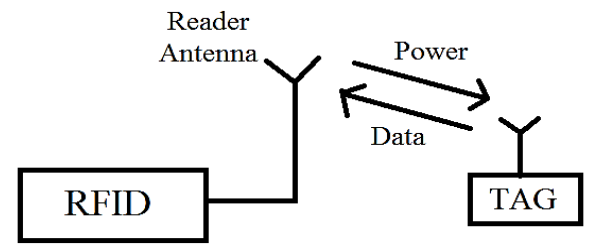

Figure 5. RFID block diagram
Table 2. RFID calculation for various slots

\begin{tabular}{ccc}
\hline Car & Voltage level & Constant value \\
\hline Car_1 & 0.37 & 1023 \\
Car_2 & 0.74 & 2046 \\
Car_3 & 1.11 & 3088 \\
Car_4 & 1.48 & 4111 \\
Car_1' & 1.85 & 5114 \\
Car_2 & 2.22 & 6137 \\
Car_3, & 2.59 & 7160 \\
Car_4, & 2.96 & 8183 \\
\hline
\end{tabular}

\section{STEPPER MOTOR}

A Stepper Motor is a brushless DC Motor. Its rotor rotates through angular steps in response to each input pulse received. A stepper motor consists of a rotor and a stator. The stator contains phase windings and rotor does not contain brushes and electrical windings. Stepper Motors can be used in high, low, medium torque applications. In this project, two stepper motors are used to lift the gates of entry and exit paths. 
According to the Figure 6, the motor has two phases i.e., A1, A2, B1, B2 respectively. The motor operates in both forward and reverse direction. The forward direction movement is via $A 1 \rightarrow B 2 \rightarrow A 2 \rightarrow B 1$. The reverse movement is $\mathrm{A} 1 \rightarrow \mathrm{B} 1 \rightarrow \mathrm{A} 2 \rightarrow \mathrm{B} 2$. The forward movement is for closing the gate and reverse movement for opening the gate. As two stepper motors are used, each phase i.e., A1,A2,B1,B2 are given some tags as q0.0, $\mathrm{q} 0.1, \mathrm{q} 0.2$, q0.3 and $\mathrm{q} 0.4, \mathrm{q} 0.5, \mathrm{q} 0.6, \mathrm{q} 0.7$ for motor 1 and 2 respectively where $\mathrm{q}$ represents the output in PLC software.

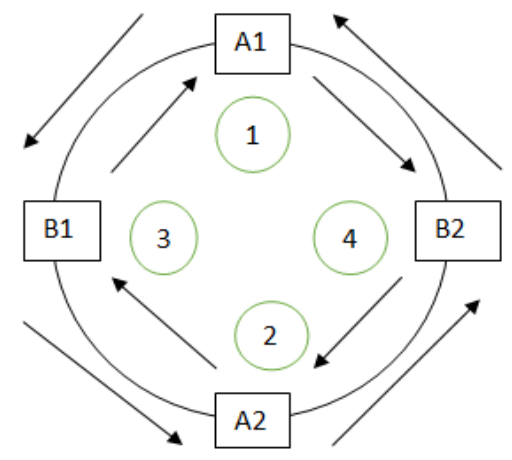

Figure 6. Operation of stepper motor

\section{HARDWARE IMPLEMENTATION}

Figure 7 shows the schematic connection of the hardware components designed in Proteus Design Suite Software. The signals from the IR sensors of all the 4 parking areas are sent to the PLC kit. Also, the signal from the RFID is sent to the microcontroller, which in turn sends it to PLC. According to the ladder logic designed in the PLC, the PLC will allow the opening and closing of the gate, which is facilitated by the motor at the entry and exit level. The command for the motor to operate is given only when the RFID detects the car and there are empty slots available based on the output of IR sensor. The corresponding motor detects the command from the PLC through the 4 motor terminals, which in turn is programmed in the PLC logic. The actual hardware implementation is shown in Figure 8.

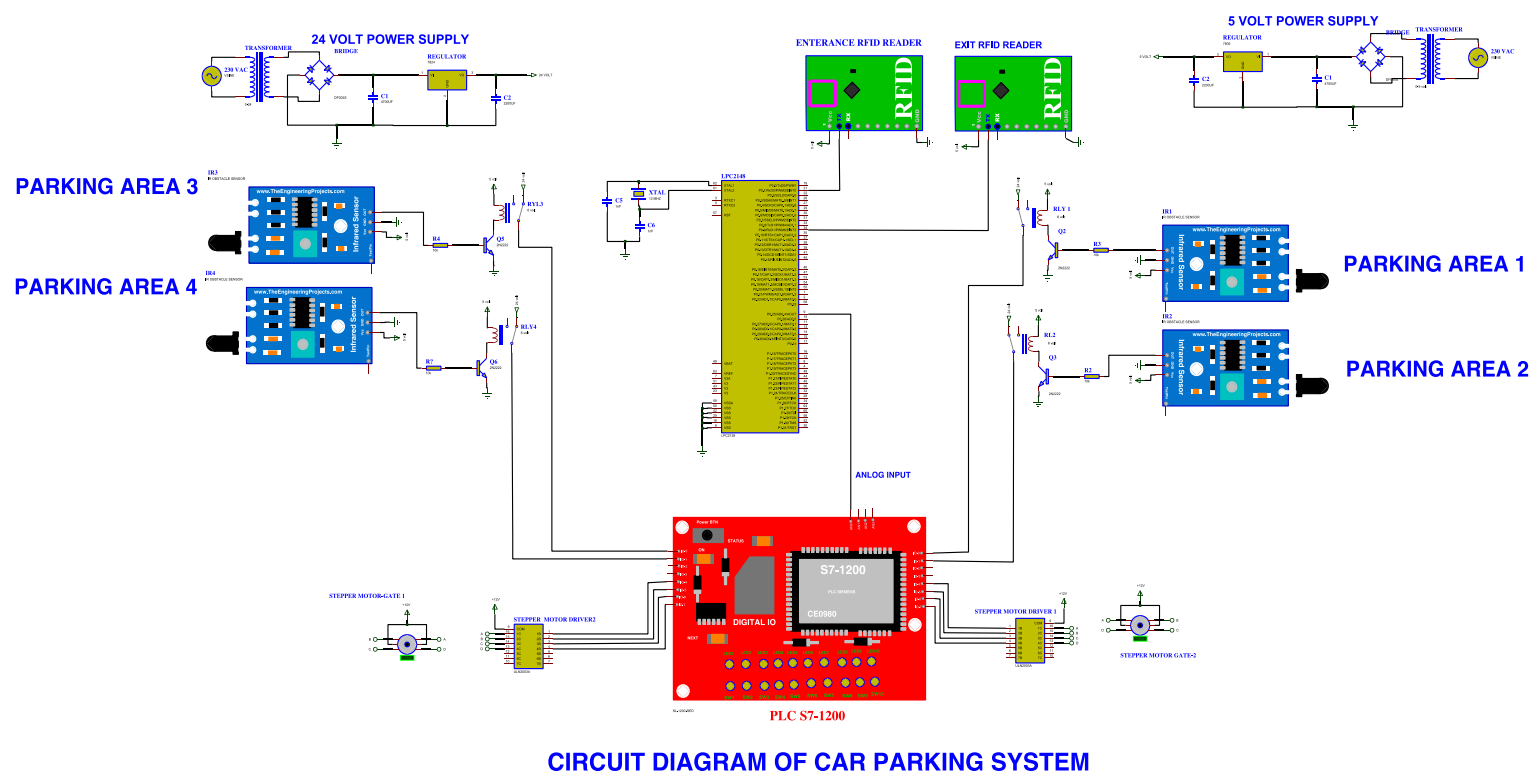

Figure 7. Hardware circuit connection 


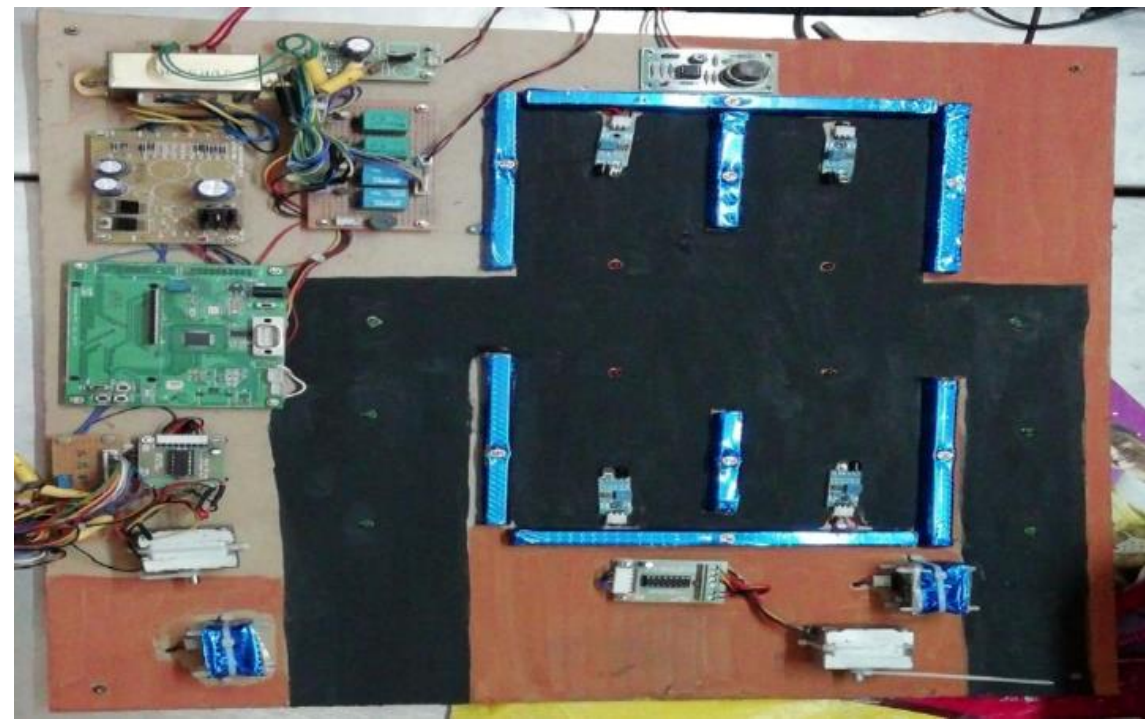

Figure 8. Hardware implementation

\section{SOFTWARE IMPLEMENTATION}

The entire software part of the project is implemented through TIA software, which supports both PLC and SCADA. We have separately designed the logic for the working of motor, RFID, car timing and the main ladder logic circuit. In Figure 9. I0.0 to I0.3 are from the IR sensors which detect the presence of car in corresponding parking slots, which in turn is given as digital inputs to the PLC. The opening of gate is designed based on the output from the IR sensors and the RFID.
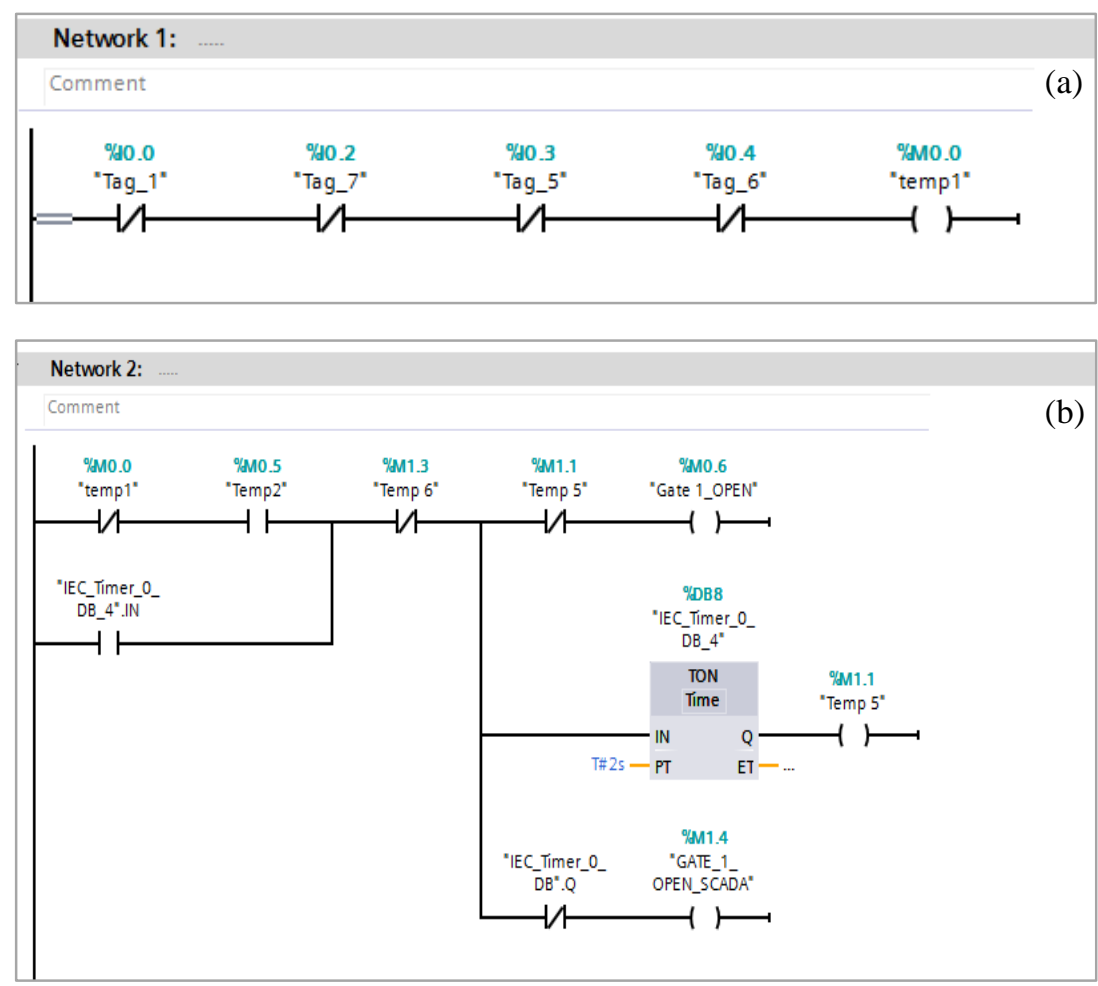

Figure 9. Main logic for parking and RFID system; (a) IR sensor, (b) RFID 

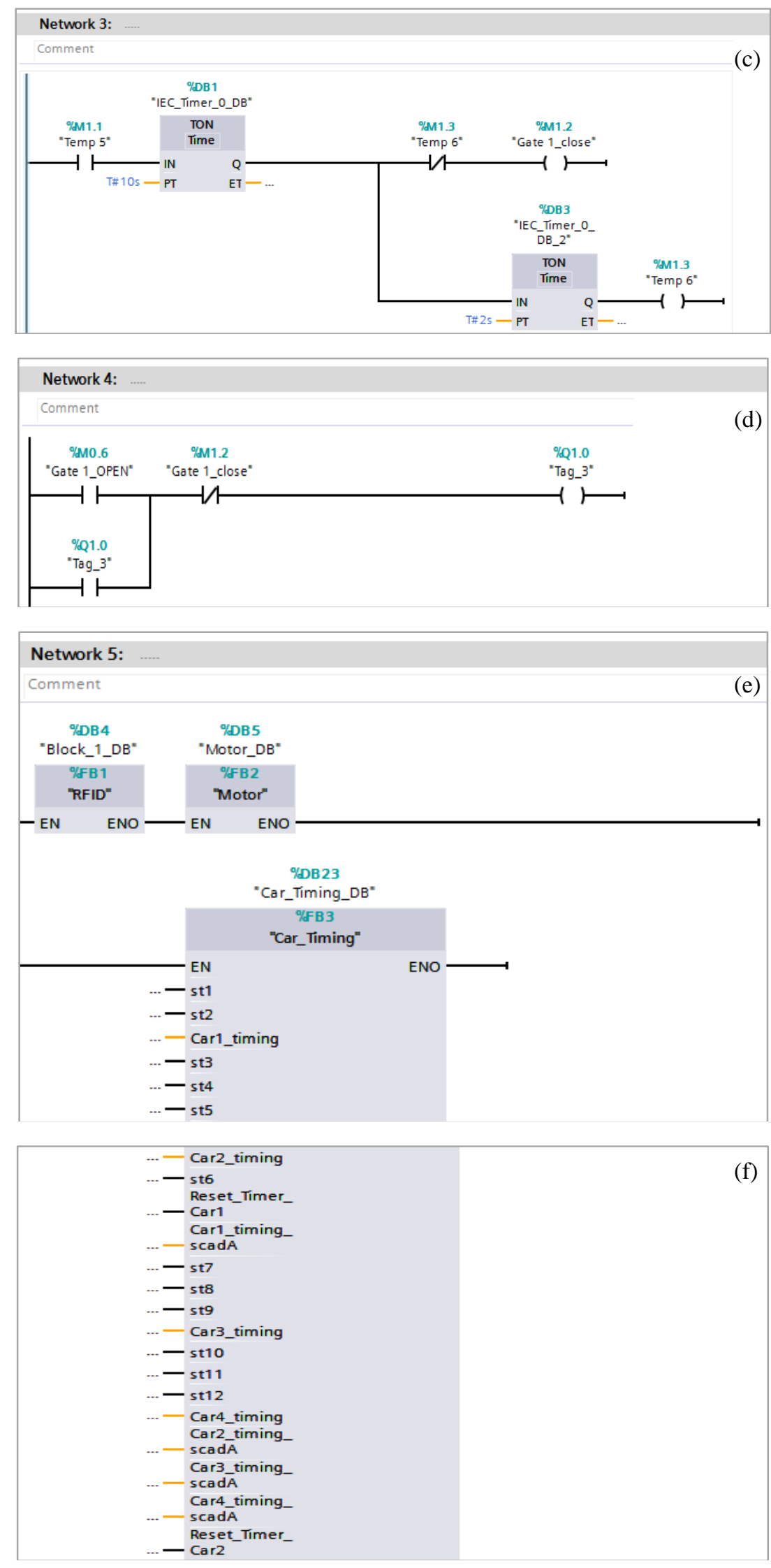

Figure 9. Main logic for parking and RFID system; (c) gate open, (d) gate close, (e) car1 timer, (f) car2 timer (continue) 

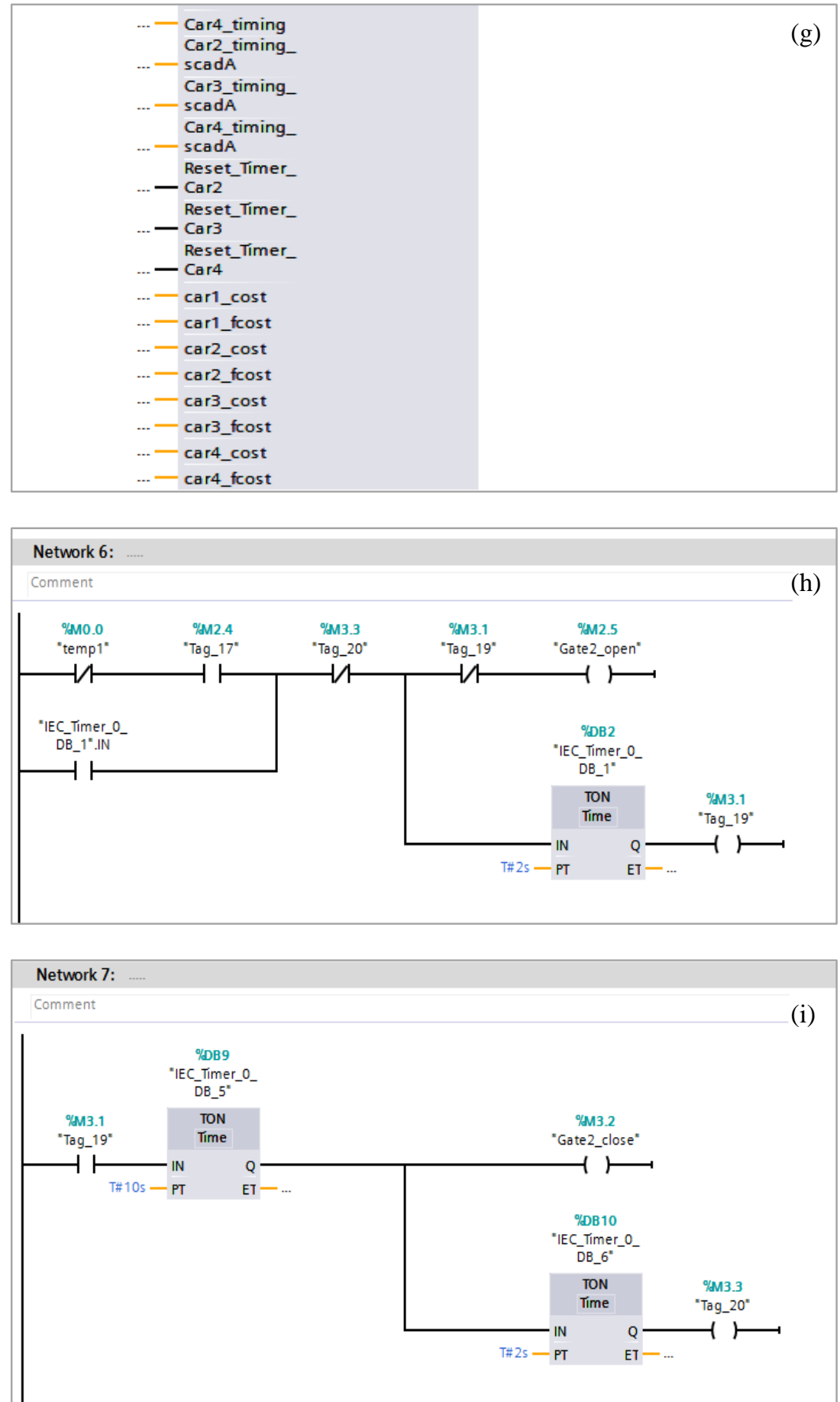

Figure 9. Main logic for parking and RFID system; (g) car3 timer, (h) gate2 open, (i) gate 2 close, (continue)

Results in the opening and closing of the gate. Similar ladder logic is designed for the $2^{\text {nd }}$ stepper motor at the exit side also. RFID logic: The ladder logic is designed based on the calculations of the constant value corresponding to a voltage level of the car. 2 comparators are used in the ladder logic for each car. Whenever the calculated value of the car lies between the comparators range it is stored in a memory. The memory for each car is connected in parallel, which means whenever any 1 of the car is detected and an empty slot is available (main logic circuit) the PLC commands the motor to operate (in the motor logic circuit). Similar logic is programmed for the RFID present at the exit side, which is shown in Figure 10. 

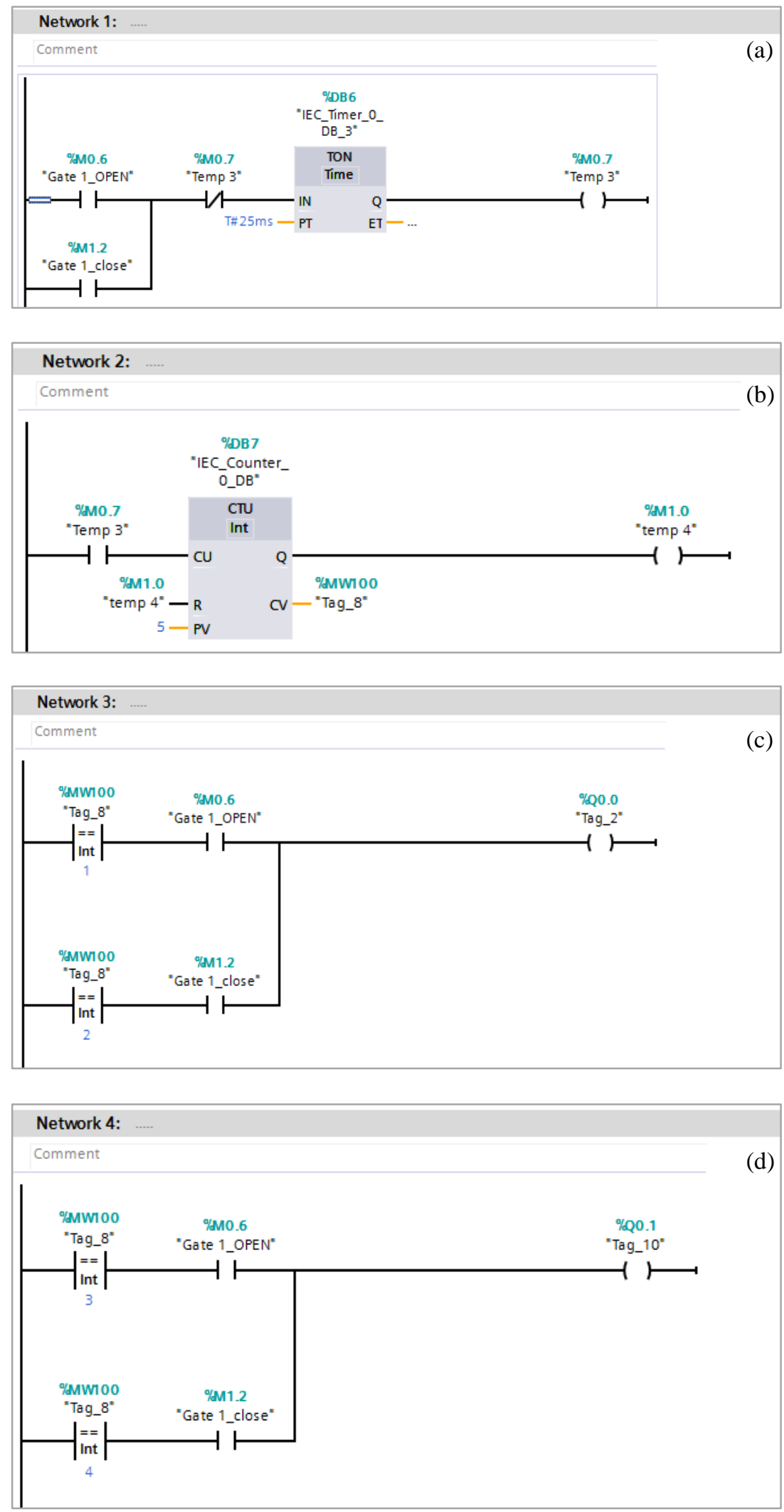

Figure 10. Motor logic of stepper motor-1; (a) open, (b) close, (c) motor ON, (d) motor OFF 


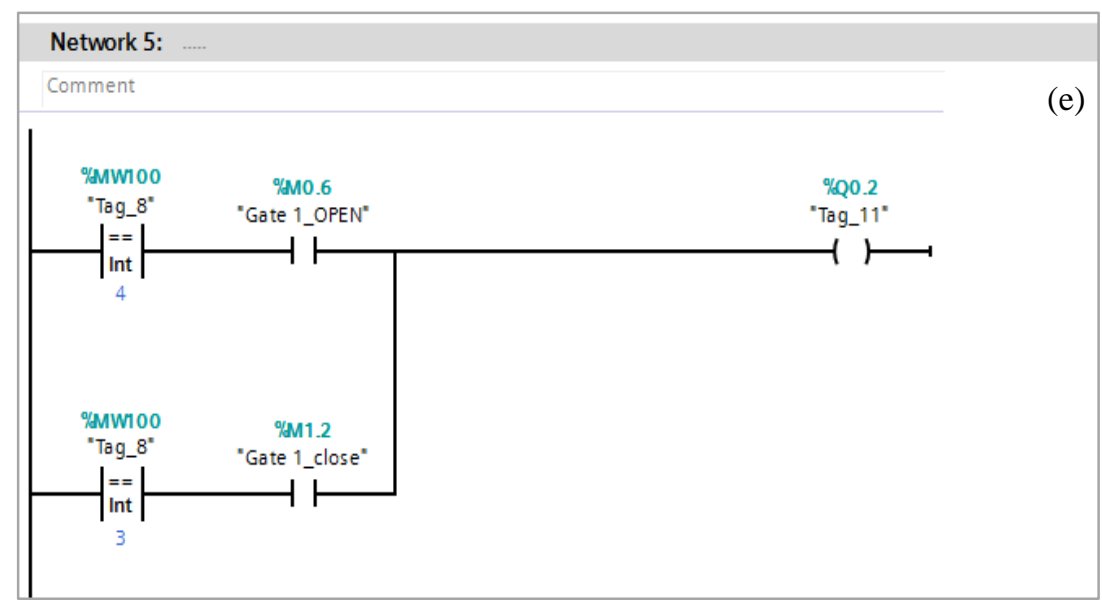

\section{Network 6:}

comment

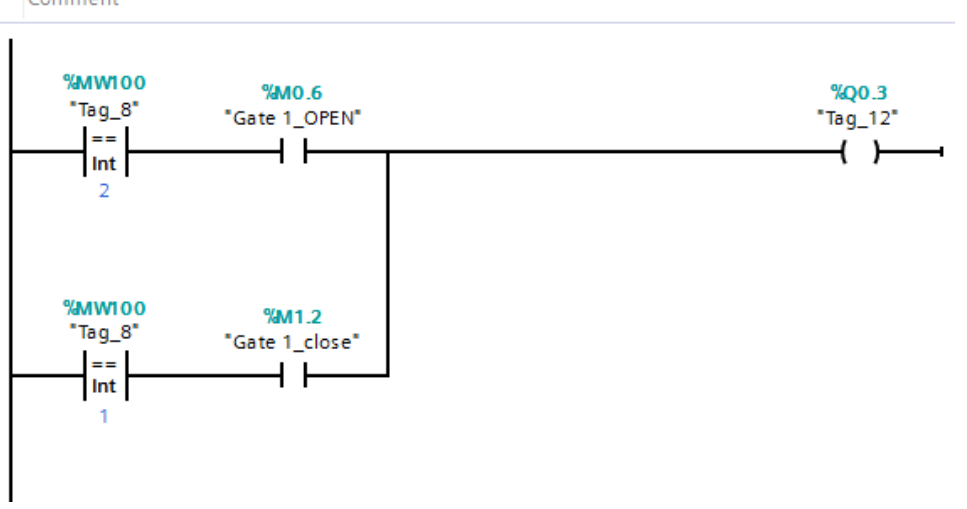

Figure 10. Motor logic of stepper motor-1; (d) gate open, (f) gate close (continue)

\section{CONCLUSION}

The project is designed and implemented to promote automation. This technique would reduce the manual effort required for such works. It will provide accurate calculation of cost of the rent based on a vehicle's stay period in the garage. Customer's safety is achieved through the installation of smoke sensors which would give signals if there happens to be any smoke in the garage. The glowing up of light only in the direction of the movement of the vehicle would assure the energy saving methodologies. This technique of vehicle monitoring, and control could be used in shopping malls, offices, colonies, schools, institutions and could also be modified for higher end usage.

\section{REFERENCES}

[1] K. Miyamoto, K. Nagano, M. Tamagawa, I. Fujita, and M. Yamamoto, "Vehicle license plate recognition by image analysis," Proceedings IECON '91: 1991 International Conference on Industrial Electronics, Control and Instrumentation, pp. 1734-1738, 1991.

[2] T. Naito, T. Tsukada, K. Yamada, K. Kozuka, and S. Yamamoto, "Robust license-plate recognition method for passing vehicles under outside environment," IEEE Transactions on Vehicular Technology, vol. 49, pp. 2309-2319, Nov 2000.

[3] H. Hontani and T. Koga, "Character extraction method without prior knowledge on size and position information," IVEC2001. Proceedings of the IEEE International Vehicle Electronics Conference 2001. IVEC 2001, pp. 67-72, 2001.

[4] J. M. Keller and R. Krishnapuram, "Fuzzy decision models in computer vision," Fuzzy Sets, Neural Networks, and Soft Computing, R. R. Yager and L. A. Zadeh, Eds. New York: Van Nostrand, pp. 213-232, 1994.

[5] D. S. Kim and S. I. Chien, "Automatic car license plate extraction using modified generalized symmetry transform and image warping," ISIE 2001. 2001 IEEE International Symposium on Industrial Electronics Proceedings, vol. 3, pp. 2022-2027, 2001.

[6] K. K. Kim, K. I. Kim, J. B. Kim, and H. J. Kim, "Learning-based approach for license plate recognition," Proc. IEEE Signal Processing Society Workshop, vol. 2, pp. 614-623, 2000. 
[7] D. M. Emiris and D. E. Koulouriotis, "Automated optic recognition of alphanumeric content in car license plates in a semi-structured environment," Proc. Int. Conf. Image Processing, vol. 3, pp. 50-53, 2001.

[8] G. Balamurugan, S. Punniakodi, K. Rajeswari, and V. Arulalan, "Automatic number plate recognition system using super-resolution technique," 2015 International Conference on Computing and Communications Technologies (ICCCT), pp. 273-277, 2015.

[9] M. V. Srinu and B. S. Shankar, "Real Time Car Parking System and Parking Fee Display Using Raspberry Pi," International Journal of Research, vol. 3, no 4, pp. 421-426, 2016.

[10] D. E. Knuth, J. Morris, James H, and V. R. Pratt, "Fast pattern matching in strings," SIAM Journal on Computing, vol. 6, no. 2, pp. 323-350, 1977.

[11] A. Broumandnia and M. Fathy, "Application of pattern recognition for Farsi license plate recognition," ICGST International Journal on Graphics, Vision and Image Processing, vol. 5, pp. 25-31, 2005.

[12] H. Saghaei, B. Seyfe, H. Bakhshi, and R. Bayat, "Novel approach to adjust the step size for closed-loop power control in wireless cellular code division multiple access systems under flat fading," IET Communications, vol. 5, no. 11, pp. 1469-1483, 2011.

[13] Hamed Saghaei, B. Seyfe, "New Approach to Closed-Loop Power Control in Cellular CDMA Systems under Multipath Fading," 2008 4th International Conference on Wireless Communications, Networking and Mobile Computing, pp. 1-4.

[14] S. L. Chang, L. S. Chen, Y. C. Chung, and S. W. Chen, "Automatic license plate recognition," IEEE Transactions on Intelligent Transportation Systems, vol. 5, no. 1, pp. 42-53, 2004.

[15] Y. Wen, Y. Lu, J. Yan, Z. Zhou, K. M. Von Deneen, and P. Shi, "An algorithm for license plate recognition applied to intelligent transportation system," IEEE Transactions on Intelligent Transportation Systems, vol. 12, no. 3, pp. 830-845, 2011.

[16] Dung L. R., Huang C. M., Wu Y. Y. "Implementation of RANSAC Algorithm for Feature-Based Image Registration," Journal of Computer and Communications, vol. 1, no. 6, pp. 46-50, 2013.

[17] Brooks R. R., Iyengar S. S., "Multi-Sensor Fusion: Fundamentals and Applications with Software," Prentice Hall, Upper Saddle River, NJ, USA, 1998.

[18] C. Yang, J. Ma, S. Qi, J. Tian, S. Zheng, and X. Tian, "Directional support value of Gaussian transformation for infrared small target detection," Applied Optics, vol. 54, no. 9, pp. 2255-2265, 2015.

[19] Palanisamy, R, Kumar, P.S, Kiran, M.P, "Arduino based accident prevention and auto intimation system," Indonesian Journal of Electrical Engineering and Computer Science, vol. 17, no. 3, pp. 1275-1280, 2020.

[20] H. Singh, V. Kumar, and S. Bhooshan, "Weighted Least Squares Based Detail Enhanced Exposure Fusion," ISRN Signal Processing, vol. 2014, 2014.

[21] Zhiqiang Zhou, Mingjie Dong, Xiaozhu Xie, Zhifeng Gao "Fusion of infrared and visible images for night-vision context enhancement," Applied Optics, vol. 55, no. 23, pp. 6480-6490, 2016.

[22] D. P. Bavirisetti and R. Dhuli, "Fusion of Infrared and Visible Sensor Images Based on Anisotropic Diffusion and Karhunen-Loeve Transform," IEEE Sensors Journal, vol. 16, no. 1, pp. 203-209, 2016.

[23] Durga Prasad Bavirisetti and Ravindra Dhuli "Two-scale image fusion of visible and infrared images using saliency detection," Infrared Physics \& Technology, vol. 76, pp. 52-64, 2016.

[24] H. Singh, H. Fatima, S. Sharma and D. Arora, "A Novel Approach for IR Target Localization Based on IR and Visible Image Fusion," 2017 4th International Conference on Signal Processing, Computing and Control (ISPCC), pp. 235-240, 2017.

[25] A. Toet, J. K. IJspeert, A. M. Waxman, and M. Aguilar, "Fusion of visible and thermal imagery improves situational awareness," Displays, vol. 18, no. 2, pp. 85-95, 1998. 